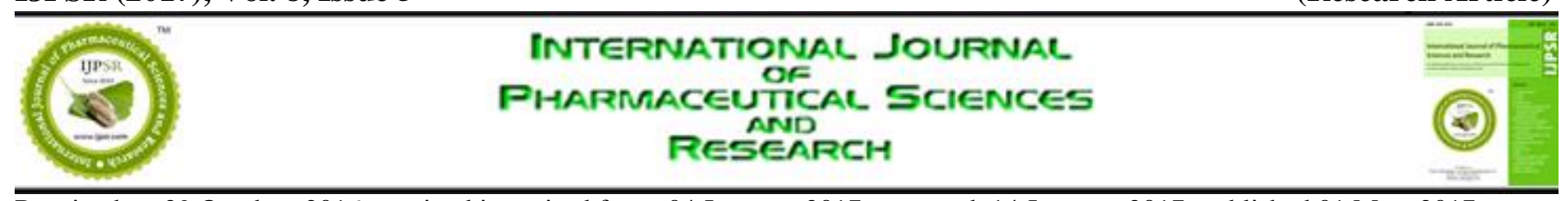

Received on 29 October, 2016; received in revised form, 04 January, 2017; accepted, 14 January, 2017; published 01 May, 2017

\title{
POLYPHARMACY AND MEDICATION COMPLIANCE IN PATIENTS WITH TYPE 2 DIABETES
}

\author{
Sehar Waheed *, Maleeha Jamal and Fatima Amin \\ Institute of Pharmacy, Lahore College for Women University, Lahore, Pakistan.
}

Keywords:

Type 2 Diabetes, Polypharmacy, Medication Compliance, Medical Regimen

\section{Correspondence to Author:}

Sehar Waheed

Student Pharm D $5^{\text {th }}$ Professional, Lahore College for Women

University, Jail Road, Lahore,

Pakistan.

Email: saherwaheed141@gmail.com

\begin{abstract}
Polypharmacy may affect patient's QOL. Polypharmacy has been shown to result in Unnecessary and/or inappropriate medication prescribing, increased risk for drug interactions and ADRs, Non adherence, and increased overall drug expenditures. That's how poly pharmacy affects patient compliance. A retrospective study was conducted about the polypharmacy and medication compliance in patient with type 2 diabetes in various teaching hospitals of Lahore. $19 \%$ of patients were prescribed at least one diabetes related medicine and $81 \%$ was prescribed more than one diabetes related medicines. 100 patients surveyed were taking a $\sum$ of 4 medicines per patient to control diabetes and related comorbidities for a total of 378 medicines. Despite the complexity of medical regimens, patients reported high level of compliance for each diabetes related medicine $92 \%$ of the prescribed doses were taken with the $6 \%$ of missing doses. It was concluded that despite the complexity of medical regimens, patients reported high level of compliance for each diabetes related medicine. Patient's perception of the immediate and future benefit of prescribed medication had a significant impact on their compliance. Moreover Attitudes and beliefs about medications play a large role in compliance.
\end{abstract}

INTRODUCTION: Polypharmacy is "the prescription, administration, or use of more medications than are clinically indicated. "Inappropriate drug combinations, unnecessary medications, and inappropriate drugs for specific patients constitute the problems of polypharmacy. Thus, patients receiving only two medications could have polypharmacy. ${ }^{1}$ Compliance describes the degree to which a patient correctly follows medical advice. Diabetes is a group of metabolic disorders characterized by hyperglycemia.

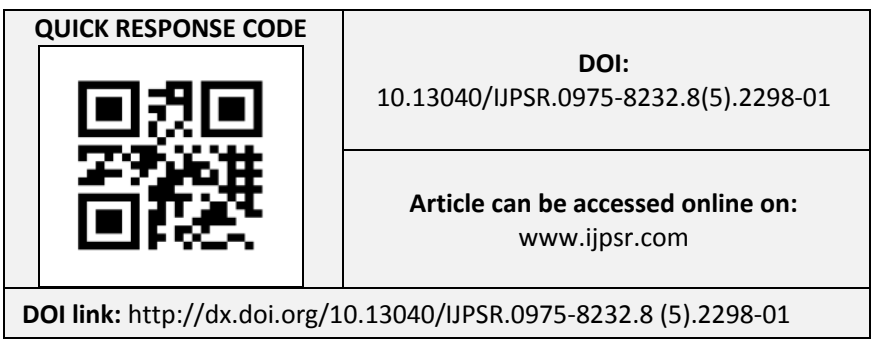

The burden of taking multiple medications has been associated with greater health care costs and an increased risk of adverse drug events druginteractions, medication non-compliance, reduced functional capacity and multiple geriatric syndromes. ${ }^{2}$ An alternative definition for polypharmacy is the use of more medications than are medically necessary. ${ }^{3}$ For this definition, medications that are not indicated, not effective, or constitute a therapeutic duplication would be considered polypharmacy. ${ }^{4}$

Patients presenting with type 2 diabetes mellitus are initially encouraged to maintain a healthy diet and exercise regimen, followed by early medication that generally includes one or more oral hypoglycemic agents and later may include an injectable treatment. To prevent the complications associated with type 2 diabetes, therapy frequently also includes medications for control of blood 
pressure, dyslipidemia and other disorders, since patients often have more than three or four chronic conditions. 5 Unfortunately, there are many negative consequences associated with polypharmacy. Specifically, the burden of taking multiple medications has been associated with greater health care costs and an increased risk of adverse drug events drug-interactions, medication non-compliance, reduced functional capacity and multiple geriatric syndromes. ${ }^{6}$ The majority of patients with type 2 diabetes fail to control glycemia with diet and exercise and require pharmacotherapy-in general, initially monotherapy with an oral hypoglycemic agents (OHA); however, owing to the progressive nature of the disease, most of the patients will eventually require combination therapy and ultimately injectable treatments as monotherapy or part of polytherapy. Glycemic control in type 2 diabetes is essential to prevent long-term micro- and macrovascular complications.

Guidelines from the ADA and the European Association for the Study of Diabetes (EASD) stress the importance of diet and exercise in the treatment of all stages of type 2 diabetes. The main reasons for non-adherence to the anti-diabetic treatment are the appointment schedule, and the exercise and diet regimen. Unavailability of transport and forgetfulness, Physician-patient relationship as well as patient's knowledge of diabetes. Even though adherence to medications leads to beneficial outcomes, it is often poor.

There are numerous reasons for poor adherence including age, social and psychological factors, education and a lack of understanding of the longterm benefits of treatment, the complexity of the medication regimen, cost of medication and negative treatment perceptions. Poor communication between doctor and patient, adverse outcomes such as weight gain and hypoglycemia, and failure of clinicians to modify medications appropriately can also affect adherence. New innovative methods are needed to assist those patients who fail in their medication compliance.

Most patients who have type 2 diabetes mellitus (DM) require several different medications. However these agents can substantially reduce diabetes-related morbidity and mortality, the extent of treatment beneficial aspects may be limited by a lack of treatment adherence.

The present study aims to determine medication compliance of patients with diabetes and to understand the impact of polypharmacy on medication compliance in different teaching hospitals of Lahore.

\section{METHODS AND MATERIALS:}

Study Area, Period and Design: A retrospective study was conducted about the polypharmacy and medication compliance in patient with type 2 diabetes. Hundred patient who have been diagnosed with type 2 Diabetes Mellitus of age 3060 years, formed the study group. Study duration was two months. Various public and private teaching hospitals were visited. A data collection form was designed and was filled during face to face interview with the patients. Data was presented in the form of graphs and tables.

RESULTS: Female patient comprised 57 (57\%) out of 100 patients surveyed with type 2 diabetes. Generic prescribing was $1.32 \%$. The average HbA1c was 9.2 with $39 \%$ patients above 9.8 and $31 \%$ below 7.8 .The average total cholesterol was $233 \mathrm{mg} / \mathrm{dl}$ with $42 \%$ above $199 \mathrm{mg} / \mathrm{dl}$. The average blood pressure was $139 \mathrm{mmHg}$ for systolic and 88 $\mathrm{mmHg}$ for diastolic. (Table 1)

\begin{tabular}{|c|c|}
\hline $\begin{array}{l}\text { TABLE 1: } \quad \text { DEMOGRAPHIC } \\
\text { PRESCRIBING INDICATORS }\end{array}$ & DATA \\
\hline Chracteristics/parameters & results \\
\hline Female patients & $57 \%$ \\
\hline Male patients & $43 \%$ \\
\hline Generic prescribing & $1.32 \%$ \\
\hline Brand prescribing & $98.68 \%$ \\
\hline Average cost per prescription & PKR.398 \\
\hline Average $\mathrm{HbA} 1 \mathrm{c}(\%)$ & 9.2 \\
\hline Patients $>9.8$ & $39 \%$ \\
\hline Patients $<7.8$ & $31 \%$ \\
\hline Average total cholesterol(mg/dl) & 233 \\
\hline Patients $>199$ & $42 \%$ \\
\hline Average blood pressure $(\mathrm{mmHg})$ & $139 / 88$ \\
\hline
\end{tabular}

Polypharmacy: $19 \%$ of patients were prescribed at least one diabetes related medicine and $81 \%$ was prescribed more than one diabetes related medicines. (Fig. 1) 


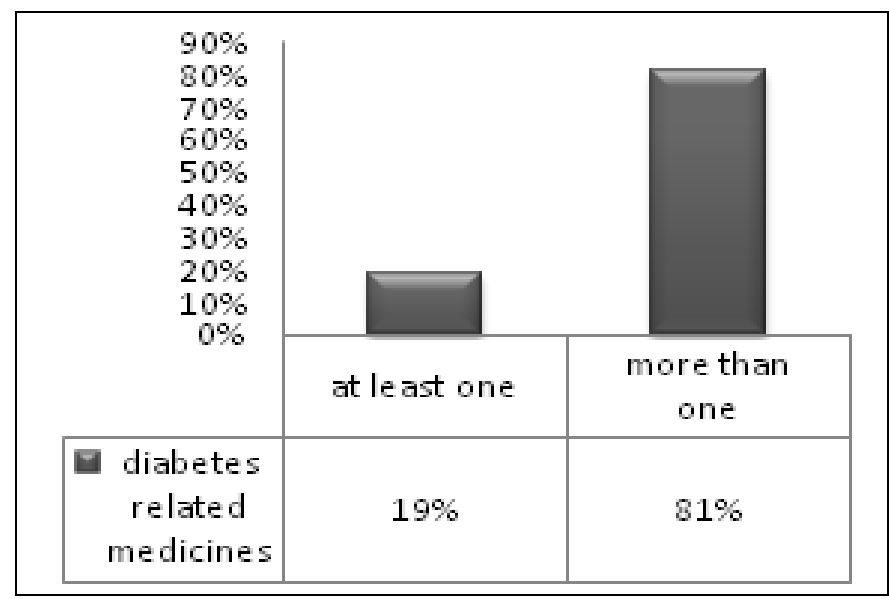

FIG. 1: DIABETES RELATED MEDICINES PRESCRIBED

$33 \%$ of patients were taking hypoglycemic agent for diabetes control, 19\% were taking insulin and $48 \%$ were taking both (hypoglycemic agent \& insulin) for diabetes control. (Fig. 2)

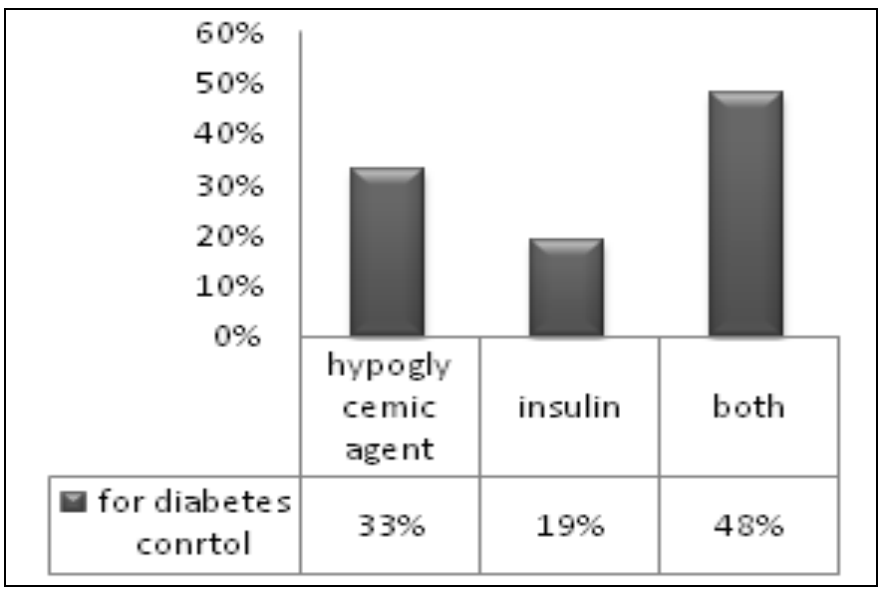

FIG. 2: MEDICATIONS RELATED TO DIABETES CONTROL

Patient Compliance: For each diabetes related medicine $92 \%$ of the prescribed doses were taken with the $6 \%$ of missing doses. (Fig. 3)

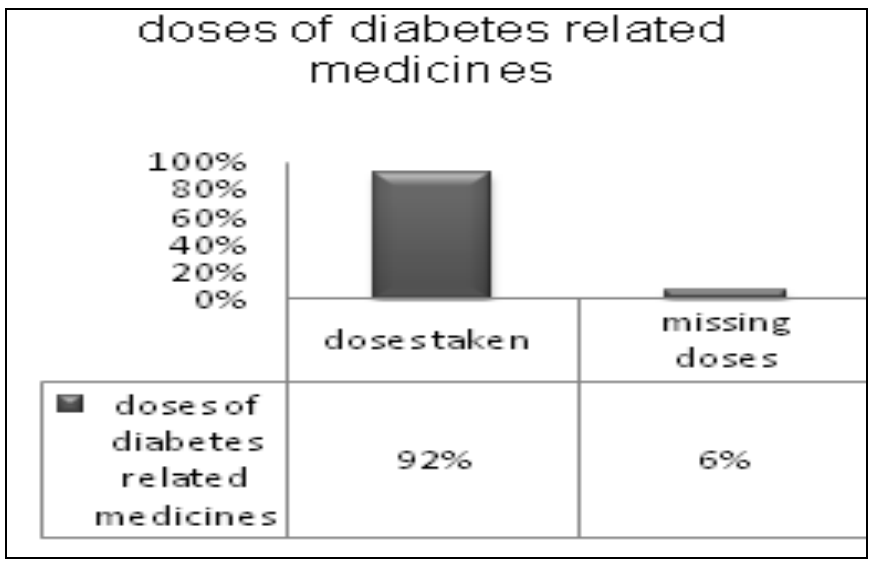

FIG. 3: DOSES TAKEN
The single most reason for having a problem taking a particular medicine was because they had no time or due to work $(33 \%)$ followed by forgot or difficulty in remembering all the doses $(27 \%)$, cost (14\%) and social reason (7\%). (Fig. 4)

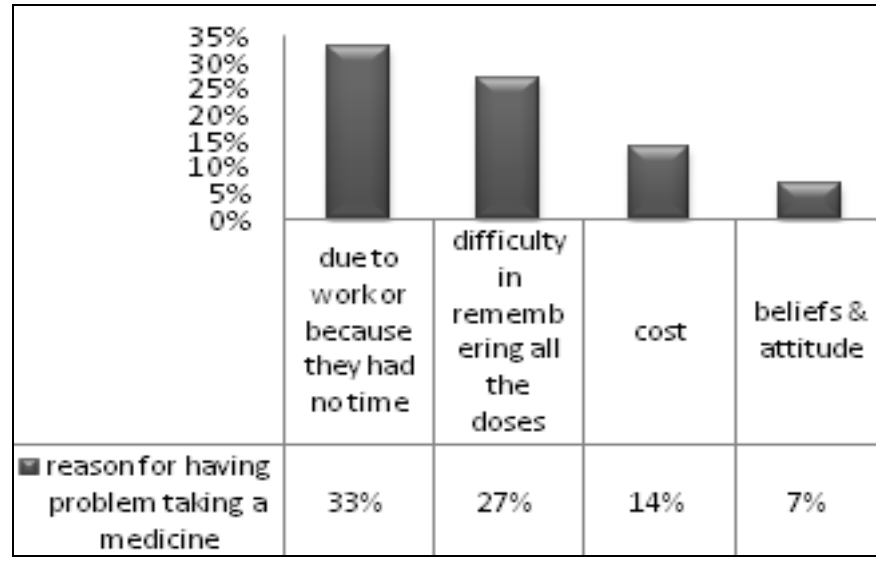

FIG. 4: REASONS FOR NON-COMPLIANCE

DISCUSSION: Polypharmacy is unavoidable, given that multiple drug therapy has become the standard of care in most chronic conditions. Poly pharmacy was observed in $81 \%$ of the patients which were prescribed with more than one diabetes related medicines including hypoglycemic agent and insulin. Most common related comorbidities were found to be hypertension, hyperlipidemia, and GI disorder, allergy, itching depression, and coagulopathies and delayed wound healing, each of which may require one or more drugs for adequate control. Thus the most commonly used therapy component used was both insulin and hypoglycemic agent. Despite the complexity of medical regimens, patients reported high level of compliance. Males showed high level of compliance then females. As in the survey females were more in number so, the most common reason for having a problem taking a particular medicine was because they had no time or due to work, followed by forgot or difficulty in remembering all the doses, cost and social reason. The lack of knowledge about prescribed medications often leads to fear of taking the medications.

CONCLUSION: Polypharmacy is unavoidable when treating a common chronic condition such as diabetes. The potential for polypharmacy will continue to increase with time, as additional therapeutic options become available. 
The most commonly used therapy component used was both insulin and hypoglycemic agent. It was concluded that despite the complexity of medical regimens, patients reported high level of compliance for each diabetes related medicine. Polypharmacy did not majorly affect the patient's compliance. Although Patient's perception of the immediate and future benefit of prescribed medication had a significant impact on their compliance. Attitudes and beliefs about medications play a large role in compliance.

ACKNOWLEDGMENT: Authors want to acknowledge this research to the university administration and hospital administration to conduct this research and helping us in every possible way.

CONFLICT OF INTEREST: The authors whose names are listed certify that they have NO affiliations with or involvement in any organization or entity with any financial interest.

\section{REFERENCES:}

1. Muir AJ, Sanders LL, Wilkinson WE, Schmader K. Reducing medication regimen complexity: a controlled trial. J Gen Intern Med. 2001; 16 (2): 77-82.

2. Robert L. Maher, Joseph T. Hanlon and Emily R. Hajjar, Expert Opin Drug Saf. 2014; 13 (1): 10.1517/14740338.2013.827660.

3. Tjia J, Velten SJ, Parsons C, et al., Studies to reduce unnecessary medication use in frail older adults: a systematic review. Drugs Aging. 2013; 30:285-307.

4. García-Pérez, LE, Álvarez, M, Dilla, T. et al., Diabetes Ther (2013) 4: 175. doi:10.1007/s13300-013-0034-y

5. American Diabetes Association. Standards of medical care in diabetes: 2013. Diabetes Care. 2013; 36 (Suppl 1): S1166.

6. Inzucchi, S.E., Bergenstal, R.M., Buse, J.B. et al., Diabetologial 2012; 55: 1577. doi:10.1007/s00125-0122534-0.s

7. http://www.ncbi.nlm.nih.gov/pmc/articles/PMC3326767/ DOR: $3 / 5 / 2016$

How to cite this article:

Waheed S, Jamal M and Amin F: Polypharmacy and medication compliance in patients with type 2 diabetes. Int J Pharm Sci Res 2017; 8(5): 2298-01.doi: 10.13040/IJPSR.0975-8232.8(5).2298-01.

All $\odot 2013$ are reserved by International Journal of Pharmaceutical Sciences and Research. This Journal licensed under a Creative Commons Attribution-NonCommercial-ShareAlike 3.0 Unported License.

This article can be downloaded to ANDROID OS based mobile. Scan QR Code using Code/Bar Scanner from your mobile. (Scanners are available on Google Playstore) 